\title{
Cemented Polyethylene Cups in Patients Younger Than 40 Years
}

\author{
Daniël C. J. de Kam MD, Jean W. M. Gardeniers MD, PhD, \\ Jan C. M. Hendriks PhD, René P. H. Veth MD, PhD, \\ B. Willem Schreurs MD, PhD
}

Received: 27 May 2008/Accepted: 1 April 2009/Published online: 16 April 2009

(C) The Author(s) 2009. This article is published with open access at Springerlink.com

\begin{abstract}
Although uncemented cup implants frequently are used in young patients, we believe long-term survival rates of cups in these patients are somewhat disappointing, and therefore we have continued to use cemented cups in primary THA, even in young patients. However, in cases of acetabular bone stock defects, we also use bone impaction grafting. We prospectively followed 130 patients with 175 cemented cups; no patients were lost to followup. The mean age of the patients at surgery was 31 years (range, 16-39 years). An acetabular reconstruction with bone impaction grafting was performed in 84 hips $(48 \%)$. The minimum followup was 2 years (average, 8.1 years; range, $2.0-18.5$ years). Twenty-one of the 175 cups (12\%) were revised at an average of 8.1 years (range, 2.0-18.5 years). Reasons for revision were infection (one early, seven late), recurrent dislocations (two), traumatic loosening (one), and aseptic loosening (10). The 10-year survival rate of all cemented cups with end point of revision for any cause was
\end{abstract}

One or more of the authors (BWS, JWMG) have received funding from Stryker-Howmedica, Newbury, UK. All received financial support was directed solely to a research fund and no personal benefits were received.

Each author certifies that his or her institution has approved the human protocol for this investigation and that all investigations were conducted in conformity with ethical principles of research.

D. C. J. de Kam, J. W. M. Gardeniers, R.

P. H. Veth, B. Willem Schreurs ( $\square)$

Department of Orthopaedic Surgery 357, Radboud University

Nijmegen Medical Centre, PO Box 9101, 6500 HB Nijmegen, the Netherlands

e-mail: b.schreurs@orthop.umcn.nl

J. C. M. Hendriks

Department of Epidemiology, Biostatistics and Health

Technology Assessment, Radboud University Nijmegen Medical

Centre, Nijmegen, the Netherlands
$85 \%$. Survival with end point of aseptic loosening of all cups was $92 \%$. Survival with end point of revision for aseptic loosening was $90 \%$ for the cups without impaction grafting and $95 \%$ for the cups with impaction grafting. We believe cemented acetabular cups in young patients have acceptable midterm survival; however, in the case of acetabular bone defects, we recommend reconstruction with impaction grafting.

Level of Evidence: Level III, therapeutic study. See the Guidelines for Authors for a complete description of levels of evidence.

\section{Introduction}

Obtaining a satisfying long-term survival of THAs in patients younger than 40 years remains a challenge. Young patients must function longer with their THA than the typical patient who has a THA, and they also engage in a higher level of activity, which is associated with higher revision rates $[19,27]$. Therefore, this population is more dependent on durable implants with excellent long-term survival. Although stem survival is acceptable in most studies, in general, cup survival is the weakest link in patients younger than 40 years $[5,8,9,15,18,20,25]$. The difference in reported survival rates between the cup and stem varies from $1 \%$ (97\% [stem] versus $96 \%$ [cup] [18]) to $11 \%$ (98.3\% [stem] versus $87.6 \%$ [cup] [20]). Despite attempts to improve cup designs and using new materials in THA, the acetabular component still shows lower survival rates than femoral implants.

One popular option is to implant uncemented acetabular cups in young patients as part of a total uncemented THA or hybrid THA (uncemented cup, cemented stem). Although cement in young patients commonly is not used 
$[1,24,35]$, we always have implanted cemented cups in patients of all ages, but with one substantial modification: in all patients with substantial acetabular bone stock deficiencies, we have reconstructed this bone stock loss using impaction bone grafting with a cemented cup. Secondary osteoarthritis resulting from underlying diseases in these young patients often is seen with associated loss of acetabular bone stock (for example, in developmental dysplasia of the hips and juvenile rheumatoid arthritis).

With this approach using cemented cups in young patients for many years, we asked whether there were any differences between cemented cups in young patients (younger than 40 years) with and without reconstruction with impaction grafting concerning (1) clinical scores, (2) revisions, (3) complications, (4) radiographic appearances, (5) polyethylene wear, and (6) survival.

\section{Materials and Methods}

We retrospectively reviewed prospectively collected data of all 130 patients (175 hips) who had a primary THA in our department between January 1988 and July 2004 and who were younger than 40 years at the time of index surgery. We used a cemented femoral stem and cemented acetabular polyethylene cup in all patients. In patients with acetabular bone deficiencies, these deficiencies were reconstructed with the impaction grafting technique. The decision to use bone impaction grafting was made based on the preoperative radiographs in combination with intraoperative findings. A trial cup was placed on the transverse ligament; in the case of a protrusion hip or a superolateral rim defect, a reconstruction was performed. Eighty-four hips (48\%) had impaction grafting whereas 91 (52\%) did not have impaction grafting. Because a cemented THA was our only treatment technique, patients with all diagnoses were included (Table 1). The majority (62\%) of the patients had developmental dysplasia of the hips, rheumatoid arthritis, or corticosteroid-induced avascular necrosis. Fifty-five (42\%) patients were males and 75 $(58 \%)$ were females. Eighty-nine $(51 \%)$ THAs were on the left side and $86(49 \%)$ were on the right. Forty-five (35\%) patients had bilateral THAs. The average age of the patients at index surgery was 31.3 years (range, 1639 years). The mean body mass index was 25.5 (range, 17.9-36.3). According to the classification of Charnley [7], 46 hips were in Category A, 71 in B, and 58 in C. We followed all patients in this prospective cohort on a regular basis and the minimum followup was 2 years (average, 8.1 years; range, 2.0-18.5 years) after surgery. During followup, six patients (eight hips) died of causes not related to the hip or hip surgery. All patients who died were
Table 1. Indications for primary THA with and without reconstruction with bone impaction grafting

\begin{tabular}{|c|c|c|c|}
\hline \multirow[t]{2}{*}{ Indication } & \multicolumn{3}{|l|}{ Number of hips } \\
\hline & $\begin{array}{l}\text { Without bone } \\
\text { impaction } \\
\text { grafting }\end{array}$ & $\begin{array}{l}\text { With bone } \\
\text { impaction } \\
\text { grafting }\end{array}$ & Total \\
\hline $\begin{array}{l}\text { Developmental dysplasia } \\
\text { of the hip }\end{array}$ & 10 & 32 & 42 \\
\hline Rheumatoid arthritis & 17 & 10 & 27 \\
\hline Perthes' disease & 4 & 4 & 8 \\
\hline $\begin{array}{l}\text { Avascular necrosis of } \\
\text { unknown cause }\end{array}$ & 6 & 2 & 8 \\
\hline Epiphyseal dysplasia & 5 & 2 & 7 \\
\hline Posttraumatic osteoarthritis & 2 & 4 & 6 \\
\hline Bechterew's disease & 3 & 2 & 5 \\
\hline Posttraumatic avascular necrosis & 4 & 1 & 5 \\
\hline Morquio's disease & 1 & 3 & 4 \\
\hline Epiphysiolysis & 1 & 3 & 4 \\
\hline Septic coxitis & 2 & 1 & 3 \\
\hline Protrusio acetabuli & 0 & 3 & 3 \\
\hline Osteomyelitis & 0 & 3 & 3 \\
\hline $\begin{array}{l}\text { Spontaneous fusion of the } \\
\text { hip of unknown cause }\end{array}$ & 1 & 1 & 2 \\
\hline Osteogenesis imperfecta & 0 & 2 & 2 \\
\hline $\begin{array}{l}\text { Polycystic disease of } \\
\text { unknown cause }\end{array}$ & 2 & 0 & 2 \\
\hline Psoriatic arthritis & 0 & 1 & 1 \\
\hline Gigantism of unknown cause & 0 & 1 & 1 \\
\hline Pseudohypoparathyroidism & 1 & 0 & 1 \\
\hline Monoarthritis of unknown cause & 0 & 1 & 1 \\
\hline $\begin{array}{l}\text { Alcohol-induced avascular } \\
\text { necrosis }\end{array}$ & 1 & 0 & 1 \\
\hline $\begin{array}{l}\text { Corticosteroid-induced } \\
\text { avascular necrosis }\end{array}$ & 31 & 8 & 39 \\
\hline Systemic lupus erythematosus & & & 9 \\
\hline $\begin{array}{l}\text { Kidney transplantation/ } \\
\text { nephropathy }\end{array}$ & & & 7 \\
\hline Subarachnoid hemorrhage & & & 4 \\
\hline Non-Hodgkin's lymphoma & & & 3 \\
\hline Crohn's disease & & & 3 \\
\hline Cerebral aneurysm & & & 2 \\
\hline Head trauma & & & 2 \\
\hline Thrombocytopenia & & & 2 \\
\hline $\begin{array}{l}\text { Hypothalamus hormone } \\
\text { substitution }\end{array}$ & & & 1 \\
\hline Germ cell tumor & & & 1 \\
\hline Aplastic anemia & & & 1 \\
\hline Pituitary adenoma & & & 1 \\
\hline Wegener's disease & & & 1 \\
\hline Acute lymphatic leukemia & & & 1 \\
\hline Meduloblastoma & & & 1 \\
\hline Total & 91 & 84 & 175 \\
\hline
\end{tabular}


followed on a regular basis and their data included; none had revision surgery. Of the original group of 175 cups, the data of only one patient were incomplete. Based on a telephone interview, the prosthesis of this patient functioned well; however, a recent radiograph was missing.

We categorized acetabular defects in accordance with the classification system of the American Academy of Orthopaedic Surgeons [10]. Eighty-six hips (49\%) had an acetabular deficiency. Type I segmental deficiencies occurred in 16 hips, Type II cavitary defects in 39 hips, and Type III combined deficiencies in 29 hips. One patient (two hips) had ankylosis of the hips, a Type V deficiency. Using impaction grafting, we reconstructed all deficiencies, including mild cavitary defects; however most were larger defects

Differences between the two groups (with and without impaction grafting) were analyzed regarding diagnosis and gender (chi square test, both $\mathrm{p}=0.001$ ). In the group with an acetabular reconstruction, a larger proportion was female and was diagnosed with developmental dysplasia of the hips compared with the group without reconstruction. There were no differences regarding age at surgery, side, bilateral THAs, followup, type of cup used, cup inner diameter, and body mass index between the two groups.

Two-thirds of the operations $(67 \%)$ were performed by or under the supervision of two senior faculty orthopaedic surgeons (BWS, JWMG). A posterolateral approach without trochanteric osteotomy was used in all hips, with the exception of two. Intraoperatively, in one patient, a preplanned Sugioka procedure was converted to a THA; however, a trochanteric osteotomy already had been performed. In the other patient, a trochanteric osteotomy was performed in a technically demanding hip with a short femoral neck. In one patient, an additional anterior approach was needed because of ankylosis of the hip. All acetabular deficiencies were reconstructed (with the exception of one case) with impaction grafting using autografts and/or allografts in 84 hips (48\%); this technique has been described in detail [28-30]. Segmental bone defects first were reconstructed with wire meshes before the morselized bone graft was impacted and a conventional full polyethylene cup was cemented. In one patient, we reconstructed a lateral rim deficiency without impaction grafting using a solid autograft fixed with two screws. In one of the ankylosed hips (Type V deficiency), we did not use impaction grafting. We used allografts only with impaction grafting in four hips $(4.8 \%)$, autografts only in 72 hips $(85.7 \%)$, and combined allografts and autografts in eight hips $(9.5 \%)$. Allografts were used when the original femoral head was not large enough to reconstruct the defect or in cases with pathologic femoral heads (for example, avascular necrosis of the femoral head). In three cases, instead of a solitary metal mesh, a solid fragment was used in combination with impaction grafting. In two of these cases, a minor segmental defect in the medial wall was closed using a cortical-trabecular fragment of a femoral head. A wire mesh was placed medial on top of the fragment and the acetabulum was reconstructed with impaction grafting. In the third case, a cortical head fragment was used to support the anterior rim together with a rim mesh in a reconstruction. The number of femoral heads used as grafts varied from one to four. In 40 hips (48\%), metal wire mesh was used for acetabular reconstruction with impaction grafting (10 medial wall meshes, 39 rim meshes). In nine early cases, we placed a mesh on top of the bone graft just before cementation, but this mesh was not part of a segmental defect reconstruction. However, after we realized this mesh did not add any stability to the reconstruction and there were no signs of damaging of the graft or graft healing by direct contact with cement, we abandoned the use of a mesh for this purpose.

We used 79 (45\%) Exeter $^{\mathrm{TM}}$ Contemporary ${ }^{\mathrm{TM}}$ cups with an inner diameter of $28 \mathrm{~mm}(\mathrm{n}=75)$ and $22.225 \mathrm{~mm}$ $(\mathrm{n}=5)$ (Stryker Howmedica, Newbury, UK), 71 (41\%) Charnley ${ }^{\circledR}$ Elite $^{\mathrm{TM}}$ cups with an inner diameter of $22.225 \mathrm{~mm}(\mathrm{n}=6)$ or $28 \mathrm{~mm}(\mathrm{n}=65)$ (DePuy, Leeds, UK), and 25 (14\%) Müller/AlloPro cups with an inner diameter of $32 \mathrm{~mm}(\mathrm{n}=19), 28 \mathrm{~mm}(\mathrm{n}=2)$, or $22.225 \mathrm{~mm}(\mathrm{n}=4)$ (Sulzer, Winterthur, Switzerland). For the femoral component, we used an Exeter ${ }^{\mathrm{TM}}$ stem in 111 cases, a Charnley ${ }^{\circledR}$ Elite $^{\mathrm{TM}}$ stem in 48 cases, and a Müller stem in 16 cases. All femoral heads used were made of a cobalt-chrome alloy; no ceramic implants were used.

We cemented acetabular components with a third-generation cementing technique. In the directly cemented cups, after reaming, multiple small drill holes were made with a 2.6-mm drill. After using pulse lavage, vacuum-mixed cement was injected directly from the cement gun and the cement was pressurized by a seal. In cases of reconstruction with bone grafts, we reamed the acetabulum, made multiple drill holes in sclerotic areas, and irrigated the acetabulum. Next the bone graft was impacted. Again, vacuum-mixed cement was injected and pressurized and the cup was inserted. Before 1989, we used Palacos ${ }^{\circledR}$ bone cement (Merck, Darmstadt, Germany); however, since 1989, we have used Surgical Simplex ${ }^{\circledR}$ (Stryker Howmedica). In 165 cases (94\%), cement loaded with antibiotics was used. All patients received antibiotic prophylaxis consisting of $2 \mathrm{~g}$ cefazolin intravenously just before surgery. Other precautionary measures to prevent infections were use of an operating theater with laminar airflow and use of two pairs of sterile gloves.

Postoperatively, all patients received thrombosis prophylaxis with low-molecular-weight heparin for 6 weeks, or before 1999, with acenocoumarol (the individual dosage regimens regulated with regular coagulation tests) for 
3 months. To prevent heterotopic ossification, we used nonsteroidal antiinflammatory drugs (NSAIDs) for 7 days. In six patients in whom NSAIDs were contraindicated, we administered one dose (7 Gy) of radiotherapy 1 day postoperatively.

Patients without acetabular reconstruction were mobilized under supervision of a physiotherapist after 1 or 2 days. Full weightbearing was increased in 2 to 6 weeks with the aid of one or two crutches. The patients who underwent impaction grafting were mobilized according to a modified protocol; in the first 6 weeks, only $10 \%$ weightbearing was allowed and then 6 to 12 weeks of $50 \%$ weightbearing using two crutches was allowed. After 12 weeks, full weightbearing mobilization was allowed. Thirty-one hips had such an extensive reconstruction of major defects that several weeks of bed rest were maintained ranging from 1 to 6 weeks. We used this modified mobilization protocol to ensure graft incorporation before full weightbearing.

Routine followups were scheduled at 6 weeks; 3, 6, and 12 months; and yearly or biannually thereafter. At our outpatient clinic, student researchers not participating in the treatment performed clinical analysis using the Harris hip score [17], the Oxford Hip Questionnaire Score (since 1998) [11], and visual analog scales for pain during rest and physical activity on a scale from 0 (no pain) to 100 (unbearable pain). We report the clinical scores of all patients excluding the 21 patients whose hips were revised during followup.

All anteroposterior pelvis and lateral radiographs of all hips were analyzed on a consensus basis by two of the authors (DCJDK, BWS). Radiographic evaluation included assessment of cup position, loosening of the acetabular component, polyethylene wear, presence of osteolysis, structural quality of the bone graft, application and position of the meshes, migration, heterotopic ossification, and fracture of the cement, mesh, or prosthesis. Radiolucent lines and osteolysis were recorded according to the three acetabular zones as described by DeLee and Charnley [12]. Radiographic loosening was defined as $2 \mathrm{~mm}$ or greater demarcation in two or three zones around the acetabular component, progressive demarcation, $3 \mathrm{~mm}$ or greater component migration, $5^{\circ}$ or greater component tilting, and/ or cement or prosthesis fracture. We determined cup migration $\left(>3-\mathrm{mm}\right.$ shift in any direction or $>5^{\circ}$ tilting) in relation to the interteardrop line instead of the Kohler line [16]. Position of the cup of $45^{\circ} \pm 10^{\circ}$ was considered normal [26]. We calculated polyethylene wear using the method of Dorr and Wan [13]. All measurements were corrected for magnification. Heterotopic ossification was classified according to the system of Brooker et al. [6]. Graft incorporation was defined as the presence of the crossing of trabecular bone on the bone-graft interface on

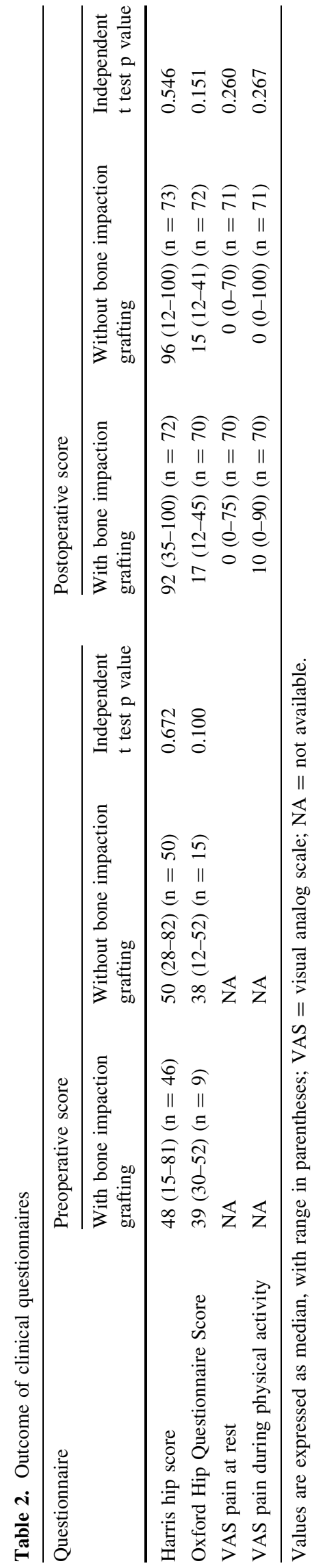


the radiographs. Clinical failure was defined as the need for revision of the acetabular component for any reason.

We calculated Kaplan-Meier curves to study the survival (time to revision). The end points were (1) cup revision for any reason, (2) cup revision for any reason excluding infections, (3) cup revision for aseptic loosening, and (4) radiographic signs of cup loosening. With an average followup of 8.1 years, $30 \%$ of all patients had a followup longer than 10 years. The log-rank test was used to test the differences in survival between cups with and without impaction grafting. Differences in outcomes between the groups were determined with the Student's ttest (continuous variables after checking for normal distribution) or chi square test (nominal variables).

\section{Results}

The outcome of the Harris hip score and the Oxford Hip Questionnaire Score improved $(\mathrm{p}<0.0001)$ after surgery for both groups; there were no differences in preoperative and postoperative clinical outcomes between the cups with and without acetabular reconstruction (Table 2). The postoperative experienced pain score was low.

The number of revisions in the groups with and without bone grafts was not different ( $\mathrm{p}=0.152)$. At last followup, 21 of the 175 cups (12\%) had been revised, seven of which had reconstruction with impaction grafting (Table 3). Reasons for revision were infection (eight), recurrent dislocations (two), traumatic loosening (one), and aseptic loosening (10). Revision for aseptic loosening was performed in 10 acetabular implants (5.7\%). The cup only was revised in eight cases and the cup and stem were revised in two cases. Four of the 10 revised cups had reconstruction with impaction grafting and six cups were implanted with standard techniques without any graft. The failed cups reconstructed with impaction grafting were revised after $4.1,9.8,16.2$, and 16.8 years (average, 11.7 years). The six directly cemented cups were revised after an average of 4.0 years (range, $1.1-10.0$ years). The time to revision for aseptic loosening was longer $(p=0.032)$ for the reconstructed cups with impaction grafting than for the cups implanted with standard techniques. The eight infected hips (4.6\%) all had revision because of culture-proven infection

Table 3. Overview of the revised cups $(n=21)$

\begin{tabular}{|c|c|c|c|c|c|c|c|}
\hline Patient & $\begin{array}{l}\text { Years to } \\
\text { revision }\end{array}$ & Cause & Part revised & $\begin{array}{l}\text { Bone } \\
\text { impaction } \\
\text { grafting }\end{array}$ & Indication & $\begin{array}{l}\text { Years to } \\
\text { radiographic } \\
\text { loosening }\end{array}$ & $\begin{array}{l}\text { Previous } \\
\text { hip operations }\end{array}$ \\
\hline 5 & 7.3 & Infection & THA & No & $\begin{array}{l}\text { Corticosteroids (systemic lupus } \\
\text { erythematosus) }\end{array}$ & & No \\
\hline 20 & 6.1 & Infection & THA & Yes & Avascular necrosis of unknown cause & & Yes \\
\hline 50 & 5.7 & Infection & THA & No & Corticosteroids (Crohn's Disease) & 2.6 & Yes \\
\hline 68 & 8.1 & Infection & THA & No & Rheumatoid arthritis & & No \\
\hline 87 & 5.3 & Infection & THA & No & Developmental dysplasia of the hip & 5.2 & Yes \\
\hline 104 & 4 & Infection & THA & No & $\begin{array}{l}\text { Corticosteroids (subarachnoid } \\
\text { bleeding) }\end{array}$ & & No \\
\hline 113 & 3.4 & Infection & THA & Yes & Corticosteroids (pituitary adenoma) & & Yes \\
\hline 123 & 2.2 & Infection & THA & No & Medial column fracture & 0.5 & Yes \\
\hline 111 & 8.6 & Recurrent dislocations & Cup & No & Posttraumatic coxarthrosis & & No \\
\hline 160 & 3.5 & Recurrent dislocations & Cup & Yes & Corticosteroids (cerebral aneurysm) & & Yes \\
\hline 84 & 10.3 & Traumatic loosening & THA & No & Corticosteroids (head trauma) & 9.9 & No \\
\hline 29 & 4.1 & Aseptic loosening & Cup & Yes & Rheumatoid arthritis & 4 & No \\
\hline 41 & 2.3 & Aseptic loosening & Cup & No & $\begin{array}{l}\text { Corticosteroids (systemic lupus } \\
\text { erythematosus) }\end{array}$ & 2.2 & Yes \\
\hline 45 & 3.1 & Aseptic loosening & Cup & No & Spontaneous fusion of unknown cause & 0.3 & No \\
\hline 49 & 1.1 & Aseptic loosening & Cup & No & $\begin{array}{l}\text { Corticosteroids (kidney } \\
\text { transplantation) }\end{array}$ & 4.2 & No \\
\hline 77 & 16.8 & Aseptic loosening & Cup & Yes & Posttraumatic coxarthrosis & 16.6 & Yes \\
\hline 78 & 9.8 & Aseptic loosening & Cup & Yes & Coxarthritis & 9.8 & Yes \\
\hline 79 & 16.2 & Aseptic loosening & THA & Yes & Developmental dysplasia of the hip & 16.2 & No \\
\hline 82 & 10 & Aseptic loosening & Cup & No & Developmental dysplasia of the hip & 5.2 & No \\
\hline 90 & 6.4 & Aseptic loosening & THA & No & Developmental dysplasia of the hip & & No \\
\hline 153 & 1.1 & Aseptic loosening & Cup & No & Epiphysiolysis & & Yes \\
\hline
\end{tabular}


of the implant. The average time to revision for septic loosening was 5.3 years (range, 2.2-8.1 years). Staphylococcus epidermidis was isolated in three, Staphylococcus aureus in two, Proprioni in two, Pseudomonas aeruginosa in one, and Streptococcus oralis in one. As a result of recurrent dislocations, two cups $(1.1 \%)$ were revised at 3.5 and 8.6 years after the index operation. One implant $(0.6 \%)$ was radiographically and clinically loose after trauma and needed revision of both components.

We observed similar $(p=0.959)$ numbers of overall complications in the groups with and without bone grafts. However, dislocations were more common $(\mathrm{p}=0.045)$ in the group without bone grafts than in the group with bone grafts (15 versus 5, respectively). Patients without reconstruction with impaction grafting had an increased dislocation chance of 1:2.9. During followup, there were nine intraoperative complications and 30 postoperative complications (Table 4). One additional stem was revised because of aseptic loosening and two femoral heads were exchanged because of recurrent dislocations. Seven hips underwent additional surgery because of postoperative complications (Table 4).

There were no differences between the cups with and without acetabular reconstruction concerning the occurrence of cup migration, radiographic loosening, or the presence of osteolysis, cysts, and abnormal cup position (Table 5). Cups with impaction grafting had fewer radiolucent lines $(p=0.02)$ and fewer lines in Zone I $(\mathrm{p}=0.001)$ (Table 5). All lines, except two, were on the bone-cement interface. In $28(48 \%)$ of the 58 cups with radiolucent lines, the lines were progressive. Of the 175 hips, 160 were radiographically stable (Fig. 1). Fifteen cups were difficult to evaluate because of overlap of the metal mesh (11 Zone I; four Zones I + II). We observed graft osteolysis in only one patient with impaction grafting; all other grafts were fully incorporated. The hip revised because of traumatic loosening had a fracture in Zone II of the acetabulum; no other fractures were seen. Fifteen (8.6\%) cups were radiographically loose, three had cup migration (after 1.8, 9.8, and 11.2 years postoperatively), and 12 had evident radiolucent lines in all zones and/or severe osteolysis; 12 of these cups were revised (Table 3).

There was no difference in polyethylene wear rates between the cups with and without impaction grafting ( $p=0.539$ in 154 unrevised cups and $p=0.525$ in the 21 revised cups) (Table 5). When looking at all cups (with and without acetabular reconstruction), the revised and radiographically loose cups had more wear compared with the cups that were not revised (both $p<0.0001$ ). Patients with an abnormal position of the cup had similar $(p=0.196)$ polyethylene wear rates to those who had a normal position. Analysis of polyethylene wear rates of cups with different inner diameters showed no differences
Table 4. Overview of complications

\begin{tabular}{|c|c|}
\hline Type of complication & Number \\
\hline \multicolumn{2}{|l|}{ Intraoperative complications $(\mathrm{n}=9)$} \\
\hline $\begin{array}{l}\text { Entrapment of sciatic nerve during } \\
\text { reposition, permanent damage }\end{array}$ & 1 \\
\hline False route femur & 1 \\
\hline Incomplete femoral fracture & 2 \\
\hline Malposition cup & 1 \\
\hline Malposition stem & 1 \\
\hline Instrument failure & 1 \\
\hline Suspicion of breakthrough of sterility & 2 \\
\hline \multicolumn{2}{|l|}{ Postoperative complications $(\mathrm{n}=30)$} \\
\hline Superficial wound infection & 3 \\
\hline Single dislocation & 9 \\
\hline Recurrent dislocations & 6 \\
\hline Sensory nerve palsy & 4 \\
\hline Sensory and motor nerve palsy & 1 \\
\hline Hematoma & 6 \\
\hline Bleeding after 4 months & 1 \\
\hline \multicolumn{2}{|l|}{ Heterotopic ossifications $(n=44)$} \\
\hline Brooker Class I & 15 \\
\hline Brooker Class II & 19 \\
\hline Brooker Class III & 10 \\
\hline \multicolumn{2}{|l|}{$\begin{array}{l}\text { Postoperative complications leading } \\
\text { to revision (no cup revision) }(\mathrm{n}=3)\end{array}$} \\
\hline Stem revision for aseptic loosening & 1 \\
\hline Head exchange because of recurrent dislocations & 2 \\
\hline \multicolumn{2}{|l|}{$\begin{array}{l}\text { Postoperative complications requiring surgical } \\
\text { intervention (no revision) }(\mathrm{n}=7)\end{array}$} \\
\hline Deep wound infection & 4 \\
\hline Heterotopic ossifications & 1 \\
\hline Traumatic dislocation & 1 \\
\hline Persistent motor and sensory nerve palsy & 1 \\
\hline
\end{tabular}

(independent $\mathrm{t}$ test, 22 versus $28 \mathrm{~mm}: \mathrm{p}=0.135,22$ versus $32 \mathrm{~mm}: \mathrm{p}=0.484,28$ versus $32 \mathrm{~mm}: \mathrm{p}=0.620$ ).

There were no differences in survival after 10 years between the groups with and without bone impaction grafting (Table 6). The midterm survival rates of all cemented polyethylene cups varied from $85 \%$ to $92 \%$ at 10 years with four end points (Table 6; Figs. 2, 3). Cup survival with an end point of radiographic loosening was $89 \%$ (95\% confidence interval, $83 \%-95 \%$ ).

\section{Discussion}

The use of cemented THA in young patients is not very popular and most surgeons will use uncemented or will resurface hips in these patients. However, we have continued to use only cemented implants in THA even in 
Table 5. Radiographic findings of all cups*

\begin{tabular}{|c|c|c|c|c|}
\hline Radiographic finding & All & $\begin{array}{l}\text { With bone } \\
\text { impaction grafting }\end{array}$ & $\begin{array}{l}\text { Without bone } \\
\text { impaction grafting }\end{array}$ & $\begin{array}{l}\mathrm{p} \text { Value (where } \\
\text { appropriate) }\end{array}$ \\
\hline Radiographic loosening & 15 & 5 & 10 & 0.608 \\
\hline Cup migration & 3 & 1 & 2 & 0.234 \\
\hline Radiolucent lines & 58 & 18 & 40 & 0.02 \\
\hline Zone I & 18 & 3 & 15 & 0.001 \\
\hline Zone II & 2 & 0 & 2 & \\
\hline Zone III & 17 & 9 & 8 & \\
\hline Zones I + II & 4 & 2 & 2 & \\
\hline Zones II + III & 5 & 2 & 3 & \\
\hline Zones I + III & 5 & 1 & 4 & \\
\hline Zones I + II + III & 7 & 1 & 6 & \\
\hline Osteolysis & 11 & 5 & 6 & 0.861 \\
\hline Zone I & 7 & 2 & 5 & \\
\hline Zone II & 1 & 1 & 0 & \\
\hline Zone III & 3 & 2 & 0 & \\
\hline Cysts & 1 & 0 & 0 & 0.033 \\
\hline Zone I & 1 & 0 & 0 & \\
\hline Zone II & 0 & 0 & 0 & \\
\hline Zone III & 0 & 0 & 0 & \\
\hline \multicolumn{5}{|l|}{ Cup position } \\
\hline Neutral position $\left(35^{\circ}-55^{\circ}\right)$ & 160 & 77 & 83 & \\
\hline Abnormal position & 15 & 7 & 8 & 0.914 \\
\hline Vertical $\left(>55^{\circ}\right)$ & 12 & 6 & 6 & \\
\hline Horizontal $\left(<35^{\circ}\right)$ & 3 & 1 & 2 & \\
\hline \multicolumn{5}{|l|}{ Polyethylene wear } \\
\hline Mean nonrevised cups (mm/year) & 0.080 & 0.076 & 0.084 & 0.539 \\
\hline Mean revised cups (mm/year) & 0.214 & 0.182 & 0.230 & 0.525 \\
\hline
\end{tabular}

* Total cups $(\mathrm{n}=175)$; Cups with $(\mathrm{n}=84)$ or without $(\mathrm{n}=91)$ reconstruction with bone impaction grafting.

young patients. In our view, the real challenge in THA in these young patients is to manage the commonly seen acetabular deficiencies. In cases of acetabular defects, we reconstruct these deficiencies with impaction bone grafting. We questioned whether there was a difference in clinical outcome, revisions, complications, radiographic appearances, polyethylene wear, and survival between the cups implanted with an acetabular reconstruction with impaction grafting and those implanted with standard cementing techniques.

Our study has several limitations: short followup, lack of assessment of activity levels, clinical interobserver variability, heterogeneous group, no comparison with other reconstruction techniques, and different types of implants used. With no patients lost to followup, our followup is representative and reliable for the midterm results [22], and long-term followup ( $>15$ years) was not available at the time of this review. Our results can be biased by an important factor we did not evaluate: the level of activity. Theoretically, with restoration of the affected hip(s) into well-functioning artificial joints, most patients will increase their level of activity. However, young patients undergoing THA with acetabular deficiencies and therefore more complex reconstructions could still have a lower level of activity after surgery relative to primary cemented cups. However, the average wear of the cups with impaction grafting was the same as the cups without impaction grafting (both $0.08 \mathrm{~mm} /$ year). Provided that activity is a major cause of polyethylene wear, this might imply the level of activity is similar in these two groups. Several studies suggest the revision and polyethylene wear rates are correlated to level of activity [2, 19, 27, 31, 38]. Additional research on level of activity and impaction grafting in young patients is necessary to confirm this hypothesis. The clinical questionnaires were obtained by student researchers who did not participate in the treatment. Multiple researchers were involved in the data collection and interobserver variability has not been tested; however, all researchers were trained and supervised to obtain these questionnaires correctly. 

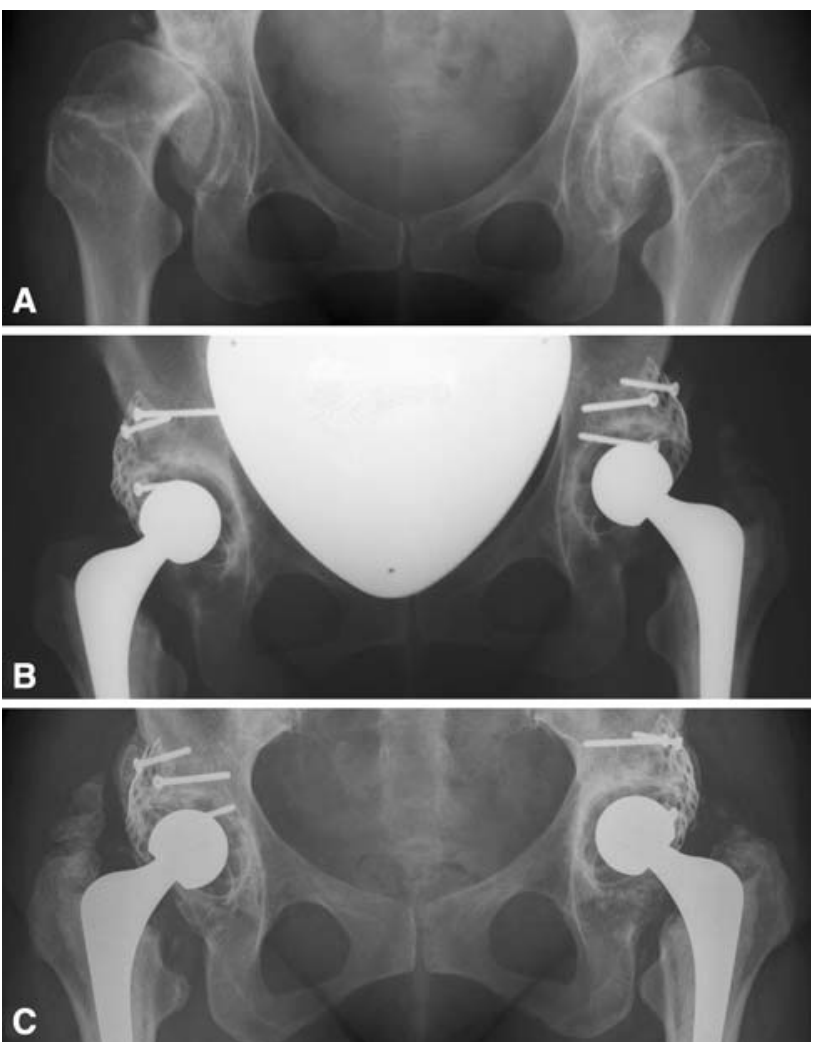

Fig. 1A-C The radiographs illustrate reconstruction of the acetabuli in a 34-year-old woman with bilateral DDH (Crowe Grade 3). (A) A preoperative anteroposterior radiograph shows the acetabuli. (B) An anteroposterior radiograph taken immediately postoperatively shows the THAs with the acetabuli reconstructed with impaction grafting. (C) An anteroposterior radiograph taken 12 years postoperatively shows the THAs remain radiographically stable, but Brooker Classes III (left) and I (right) heterotopic ossifications are visible.

The clinical scores were comparable between the two groups and comparable to published scores (Table 7). Although the cups reconstructed with bone impaction grafting were the more demanding procedures, no clinical differences were seen.

Although revision rates in both groups were comparable, the time to revision was longer in the cups reconstructed with bone impaction grafting. We have no clear explanation for this observation; possibly the cement-bone
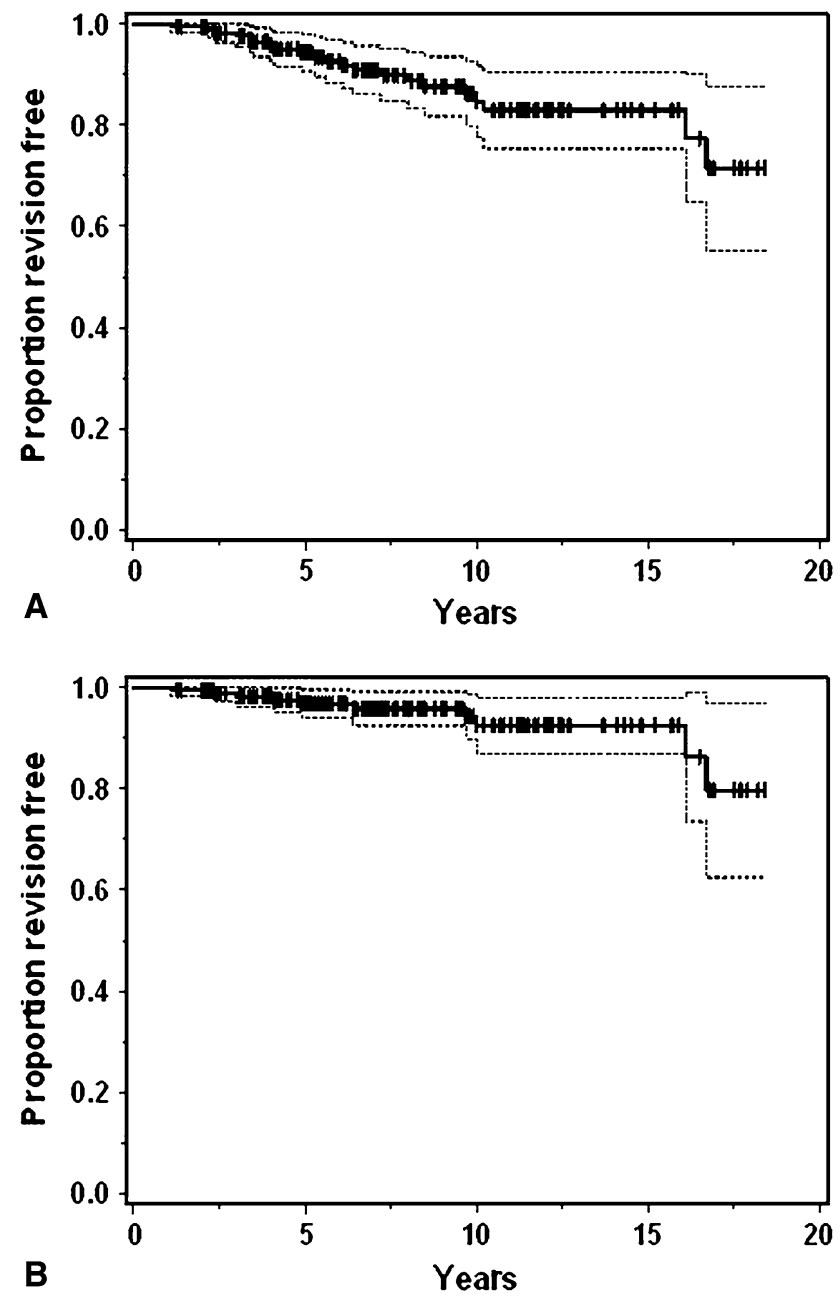

Fig. 2A-B Kaplan-Meier survival curves with $95 \%$ confidence intervals (broken lines) of all cups with end points of (A) revision for any reason and $(\mathbf{B})$ revision for aseptic loosening are shown. The vertical bars indicate the censored data points.

interface was better in cups with bone impaction grafting with better interdigitation of the cement into the bone [36]. This also may explain the lower incidence of radiolucent lines in the cups reconstructed with bone impaction grafting. The number of revisions for septic loosening was relatively high during this midterm followup study (4.6\%). Only one septic loosening likely was related to the surgery;

Table 6. The 10-year survival rates*

\begin{tabular}{|c|c|c|c|c|}
\hline End point & All cups & $\begin{array}{l}\text { Without bone impaction } \\
\text { grafting }\end{array}$ & $\begin{array}{l}\text { With bone impaction } \\
\text { grafting }\end{array}$ & $\begin{array}{l}\text { Log-rank } \\
\text { p value }\end{array}$ \\
\hline Revision for any reason & $85 \%(78 \%-92 \%)$ & $79 \%(68 \%-90 \%)$ & $91 \%(82 \%-99 \%)$ & 0.21 \\
\hline $\begin{array}{l}\text { Revision for any reason excluding } \\
\text { infections }\end{array}$ & $91 \%(85 \%-97 \%)$ & $87 \%(78 \%-99 \%)$ & $94 \%(87 \%-100 \%)$ & 0.56 \\
\hline Revision for aseptic loosening & $92 \%(87 \%-98 \%)$ & $90 \%(81 \%-99 \%)$ & $95 \%(89 \%-100 \%)$ & 0.73 \\
\hline
\end{tabular}

* Kaplan-Meier estimates; 95\% confidence interval in parentheses. 

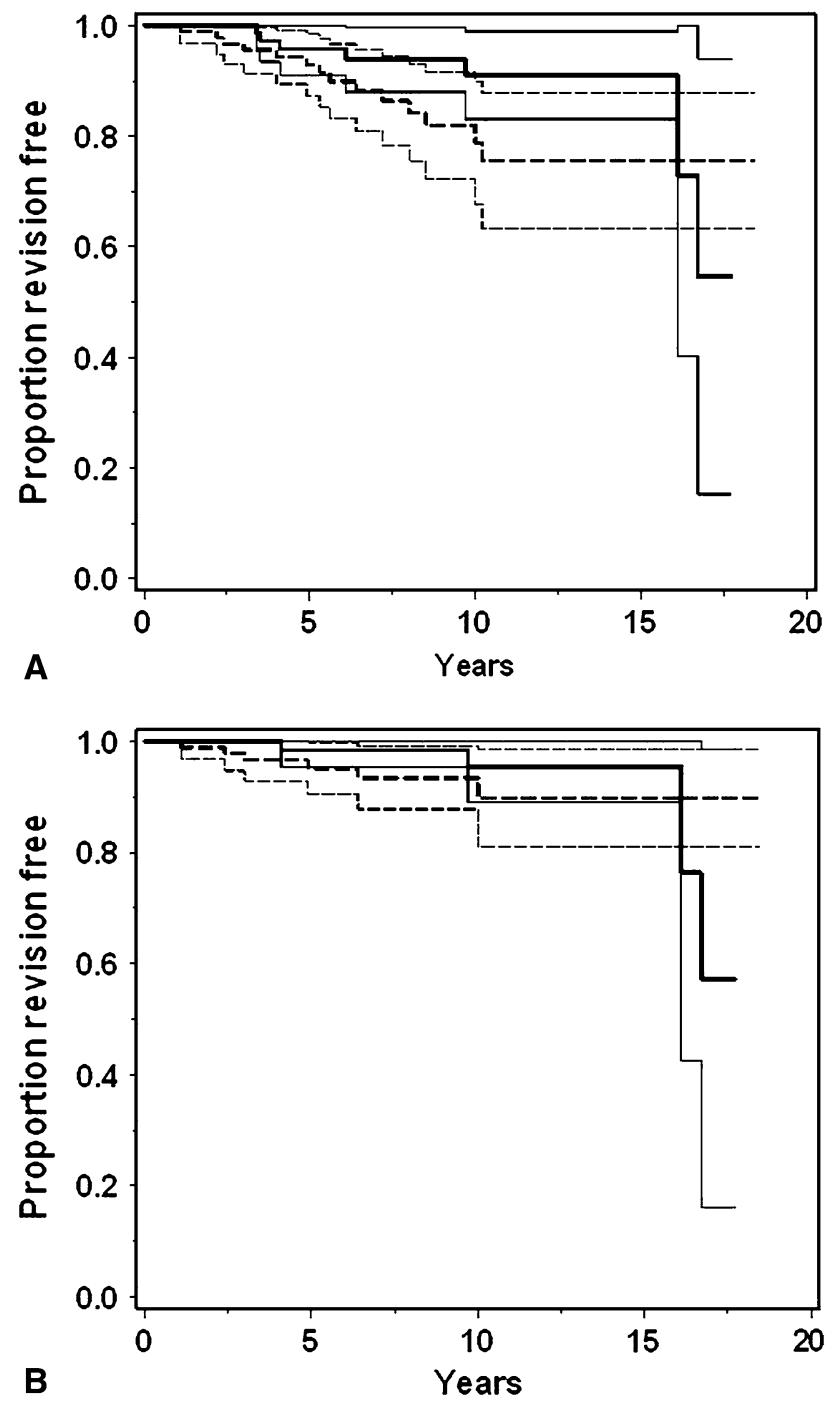

Fig. 3A-B Kaplan-Meier survival curves of cups without impaction grafting (thick broken line, $95 \%$ confidence intervals in thin broken lines) and cups with impaction grafting (thick solid line, 95\% confidence intervals in thin solid lines) with end points of (A) revision for any reason and (B) revision for aseptic loosening are shown.

we considered all other infections acute hematogenous infections of previously well-functioning prostheses. The use of corticosteroids and newer rheumatic disease- modifying drugs, which were used in most of the infection cases, can explain this higher risk of infection [3, 4]. Sochart and Porter [33] had only two infections in their study, but both were in patients with rheumatoid arthritis. Still, our revision rate for septic loosening of $4.6 \%$ is relatively high in contrast to other studies, such as that of Joshi et al. [18], with an infection rate of $1.3 \%$. Remarkably, many septic loosenings occurred late ( $>2$ years postoperatively).

The cups reconstructed with impaction grafting showed fewer complications by having fewer dislocations than the cups implanted by standard techniques. This might be attributed to the different mobilization protocol for the patients who received cups with impaction grafting. Immobilization is associated with lower dislocation rates [21]. The overall dislocation rate in our study was $11.4 \%$, which is relatively high. However, subluxation rates in young patients having THA have been reported to be as much as $18.2 \%$ [14]. The overall complication rate of $17 \%$ (30 postoperative complications) is also relatively high. Joshi et al. [18] reported a complication rate of $11.5 \%$ in cemented hips and Duffy et al. [15] reported a complication rate of $12 \%$ during the perioperative period.

As expected, revised and radiographically loose cups showed more polyethylene wear. This is consistent with previous reports showing wear particles are associated with osteolysis in THA [27, 33, 37]. The average wear rate of the cups of $0.08 \mathrm{~mm} / \mathrm{year}$ is within the normal limits, keeping in mind that wear in younger patients can be $33 \%$ to $40 \%$ higher than wear in older patients [27]. In a large study of 226 hips in patients younger than 40 years with a Charnley ${ }^{\circledR}$ prosthesis, Sochart and Porter [33] reported an average wear rate of 0.08 to $0.10 \mathrm{~mm} / \mathrm{year}$ in the nonrevised cups, which is comparable to our results. Wan et al. [37] found a correlation between inclination of the cup and higher/lower wear rates. However, we did not observe higher wear with abnormal position or inner cup diameter.

The observed overall midterm survival of cemented polyethylene cups in patients younger than 40 years in our study was acceptable. Especially in these young patients, there is a need for total hip implants with proven long-term survival [20]. Although the use of uncemented prostheses in these young patients is very popular, literature regarding

Table 7. Reported outcomes of the Harris hip score in patients $<40$ years for primary THA

\begin{tabular}{lllll}
\hline Study & Questionnaire & Preoperative score & Postoperative score & Paired t-test p value \\
\hline Chiu et al. [8] & Harris hip score & $44(26-74)$ & $88(74-99)$ & $<0.001$ \\
$\begin{array}{l}\text { Duffy et al. [15] } \\
\text { Current study }\end{array} \quad$ Harris hip score & 51 & 92 & $<0.001$ \\
$\quad$ With bone impaction grafting & Harris hip score & $48(15-81)$ & $92(35-100)$ & $<0.001$ \\
$\quad$ Without bone impaction grafting & Harris hip score & $50(28-82)$ & $96(12-100)$ & $<0.001$ \\
\hline
\end{tabular}

Values are expressed as median (current study) or means (other studies), with range in parentheses. 


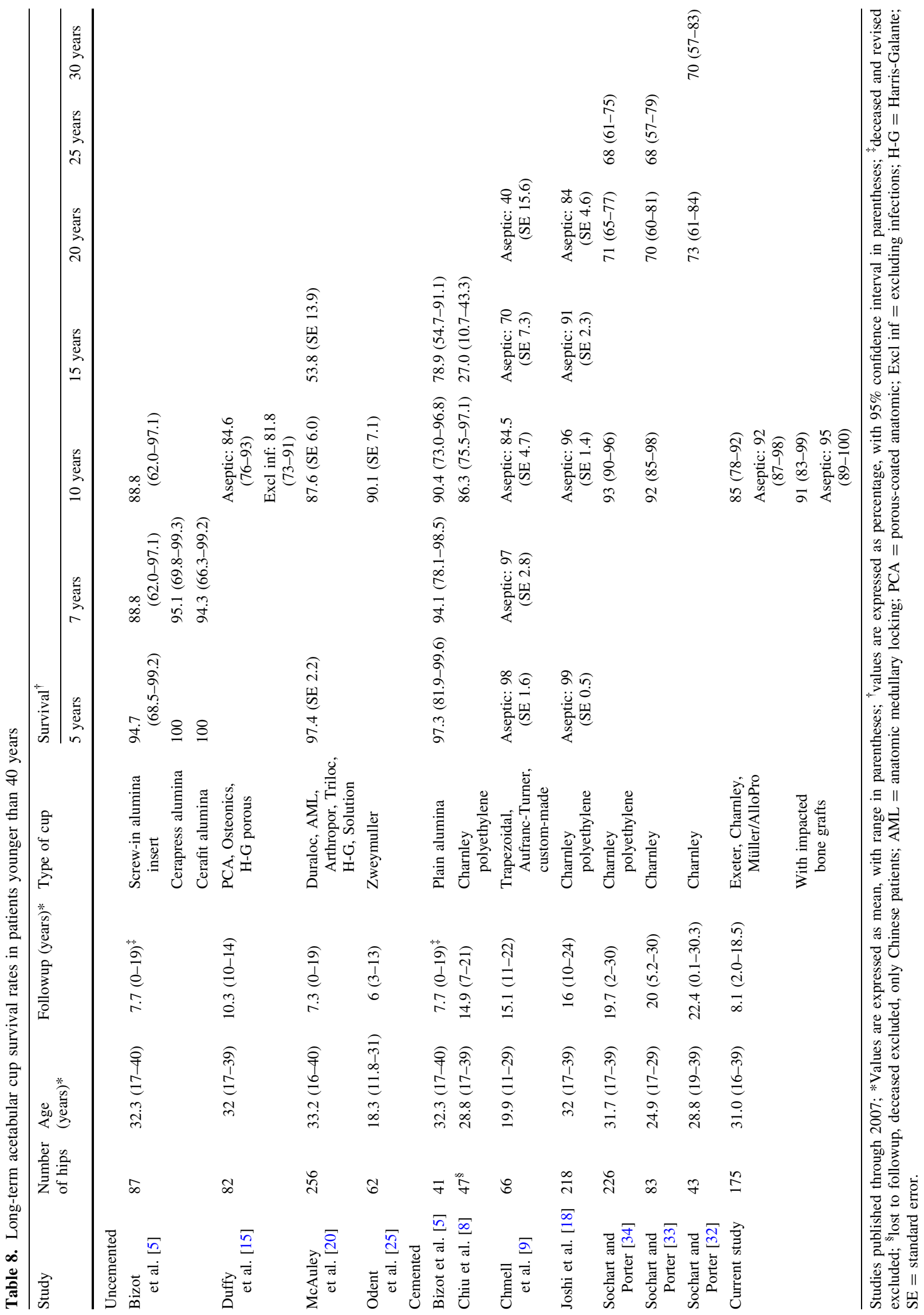


long-term outcome of THA in patients younger than 40 years concerns mainly studies of cemented implants and less about uncemented implants (Table 8) [5, 8, 9, 15, 18, $20,25,32-34]$. A limitation of the reported midterm or long-term results of uncemented cups is the fact that in these studies first-generation uncemented cups were used. The long-term outcome of improved newer uncemented cup designs remains unclear. Most of these older cup designs no longer are available. The only report of uncemented cups at 15 years after surgery with an end point of revision for any reason showed a survival rate of 54\% [20]. This is less favorable than the results of cemented cups at that time (Table 8). Sochart and Porter [34] had survival rates of $71 \%$ and $68 \%$ at 20 and 25 years, respectively, for cemented Charnley ${ }^{\circledR}$ cups. The survival of the acetabular uncemented cups with an end point of revision for aseptic loosening in patients younger than 40 years reported in one study was $85 \%$ [15], in contrast to a survival rate of $96 \%$ after 10 years of the Charnley ${ }^{\circledR}$ cups in the study by Joshi et al. [18]. We found a survival rate with cemented cups of $92 \%$ at 10 years with an end point of revision for aseptic loosening.

A remarkable finding of our study was the survival of cups with acetabular reconstructions with impaction grafting was at least comparable to the survival of standard cemented cups, especially considering the more difficult hips of our study population needed reconstruction with impaction grafting. Our data on the cemented cups with impaction grafting showed similar survival, where rather lower survival rates would be expected. The outcome of these cups reconstructed with impaction grafting even fulfilled the NICE criteria (a survival of $>90 \%$ after 10 years) [23], with a survival rate of $91 \%$ at 10 years with an end point of revision for any reason. The survival rates of the cemented cups in our study are comparable to those reported for cemented cups [8, 18, 34].

Although cemented cups are not commonly used in young patients, our data suggest cemented conventional polyethylene cups are still a good option in THA in young patients. Even reconstruction of (severe) acetabular deficiencies with impaction grafting and a cemented conventional polyethylene cup produced very acceptable survival rates, comparable to the rates of cemented cups implanted in acetabuli without deficiencies with standard cementing techniques.

Acknowledgments We thank Jaap Brunnekreef for assistance with data management.

Open Access This article is distributed under the terms of the Creative Commons Attribution Noncommercial License which permits any noncommercial use, distribution, and reproduction in any medium, provided the original author(s) and source are credited.

\section{References}

1. Australian Orthopaedic Association. National Joint Replacement Registry; Annual Report 2007. Available at: http://www.aoa. org.au/docs/njarep07.pdf. Accessed December 1, 2008.

2. Beaulé PE, Dorey FJ, Hoke R, LeDuff M, Amstutz HC. The value of patient activity level in the outcome of total hip arthroplasty. J Arthroplasty. 2006;21:547-552.

3. Berbari EF, Hanssen AD, Duffy MC, Steckelberg JM, Ilstrup DM, Harmsen WS, Osmon DR. Risk factors for prosthetic joint infection: case-control study. Clin Infect Dis. 1998;27:1247-1254.

4. Berbari EF, Osmon DR, Duffy MC, Harmssen RN, Mandrekar JN, Hanssen AD, Steckelberg JM. Outcome of prosthetic joint infection in patients with rheumatoid arthritis: the impact of medical and surgical therapy in 200 episodes. Clin Infect Dis. 2006;42:216-223.

5. Bizot P, Banallec L, Sedel L, Nizard R. Alumina-on-alumina total hip prostheses in patients 40 years of age or younger. Clin Orthop Relat Res. 2000;379:68-76.

6. Brooker AF, Bowerman JW, Robinson RA, Riley LH. Ectopic ossification following total hip replacement: Incidence and a method of classification. J Bone Joint Surg Am. 1973;55:1629-1632.

7. Charnley J. The long-term results of low-friction arthroplasty of the hip performed as a primary intervention. J Bone Joint Surg Br. 1972;54:61-76.

8. Chiu KY, Ng TP, Tang WM, Poon KC, Ho WY, Yip D. Charnley total hip arthroplasty in Chinese patients less than 40 years old. J Arthroplasty. 2001;16:92-101.

9. Chmell MJ, Scott RD, Thomas WH, Sledge CB. Total hip arthroplasty with cement for juvenile rheumatoid arthritis: results at a minimum of ten years in patients less than thirty years old. J Bone Joint Surg Am. 1997;79:44-52.

10. D'Antonio JA, Capello WN, Borden LS, Bargar WL, Bierbaum BF. Classification and management of acetabular abnormalities in total hip arthroplasty. Clin Orthop Relat Res. 1989;243:126-137.

11. Dawson J, Carr A, Murray D. Questionnaire on the perceptions of patients about total hip replacement. J Bone Joint Surg Br. 1996;78:185-190.

12. DeLee JG, Charnley J. Radiological demarcation of cemented sockets in total hip replacement. Clin Orthop Relat Res. 1976;121:20-32.

13. Dorr LD, Wan Z. Comparative results of a distal modular sleeve, circumferential coating, and stiffness relief using the Anatomic Porous Replacement II. J Arthroplasty. 1996;11:419-428.

14. Dudkiewicz I, Salai M, Blankstein A, Chechik A. Total hip arthroplasty in patients younger than 30 years of age following developmental dysplasia of hip (DDH) in infancy. Arch Orthop Trauma Surg. 2002;122:139-142.

15. Duffy GP, Berry DJ, Rowland C, Cabanela ME. Primary uncemented total hip arthroplasty in patients $<40$ years old: 10 - to 14 -year results using first-generation proximally porous-coated implants. J Arthroplasty. 2001;16:140-144.

16. Goodman SB, Adler SJ, Fyhrie DP, Schurman DJ. The acetabular teardrop and its relevance to acetabular migration. Clin Orthop Relat Res. 1988;236:199-204.

17. Harris WH. Traumatic arthritis of the hip after dislocation and acetabular fractures: treatment by mold arthroplasty. An endresult study using a new method of result evaluation. J Bone Joint Surg Am. 1969;51:737-755.

18. Joshi AB, Porter ML, Trail IA, Hunt LP, Murphy JC. Long-term results of Charnley low-friction arthroplasty in young patients. J Bone Joint Surg Br. 1993;75:616-623.

19. Kobayashi S, Eftekhar NS, Terayama K, Joshi RP. Comparative study of total hip arthroplasty between younger and older patients. Clin Orthop Relat Res. 1997;339:140-151. 
20. McAuley JP, Szuszczewicz ES, Young A, Engh CA Sr. Total hip arthroplasty in patients 50 years and younger. Clin Orthop Relat Res. 2004;418:119-125.

21. Morrey BF. Difficult complications after hip joint replacement: dislocation. Clin Orthop Relat Res. 1997;344:179-187.

22. Murray DW, Britton AR, Bulstrode CJ. Loss to follow-up matters. J Bone Joint Surg Br. 1997;79:254-257.

23. National Institute for Clinical Excellence (NICE) (2003) Guidance on the Selection of Prostheses for Primary Hip Replacement. London, UK: NICE.

24. Norwegian Arthroplasty Register. Annual Report 2007. Available at: http://www.haukeland.no/nrl/Report2007.pdf. Accessed December 1, 2008.

25. Odent T, Journeau P, Prieur A, Touzet P, Pouliquen J. Cementless hip arthroplasty in juvenile idiopathic arthritis. $J$ Pediatr Orthop. 2005;25:465-470.

26. Salvati EA, Chuem V, Aglietti P, Wilson PD. Radiology of total hip replacements. Clin Orthop Relat Res. 1976;121:74-82.

27. Schmalzried TP, Huk OL. Patient factors and wear in total hip arthroplasty. Clin Orthop Relat Res. 2004;418:94-97.

28. Schreurs BW, Busch VJ, Welten ML, Verdonschot N, Slooff TJ. Acetabular reconstruction with impaction bone-grafting and a cemented cup in patients younger than fifty years old. $J$ Bone Joint Surg Am. 2004;86:2385-2392.

29. Schreurs BW, Slooff TJ, Gardeniers JW, Buma P. Acetabular reconstruction with bone impaction grafting and a cemented cup: 20 years' experience. Clin Orthop Relat Res. 2001;393: 202-215.

30. Schreurs BW, Van Tienen TG, Buma P, Verdonschot N, Gardeniers JW. Favorable results of acetabular reconstruction with impacted morsellized bone grafts in patients younger than 50 years: a 10- to 18-year follow-up study of 34 cemented total hip arthroplasties. Acta Orthop Scand. 2001;72:120-126.

31. Sechriest VF 2nd, Kyle RF, Marek DJ, Spates JD, Saleh KJ, Kuskowski M. Activity level in young patients with primary total hip arthroplasty: a 5-year minimum follow-up. J Arthroplasty. 2007;22:39-47.

32. Sochart DH, Porter ML. Long-term results of total hip replacement in young patients who had ankylosing spondylitis: eighteen to thirty-year results with survivorship analysis. J Bone Joint Surg Am. 1997;79:1181-1189.

33. Sochart DH, Porter ML. The long-term results of Charnley lowfriction arthroplasty in young patients who have congenital dislocation, degenerative osteoarthritis, or rheumatoid arthritis. J Bone Joint Surg Am. 1997;79:1599-1617.

34. Sochart DH, Porter ML. Long-term results of Charnley lowfriction arthroplasty in patients aged less than 30 years. J Arthroplasty. 1998;13:123-131.

35. Swedish Hip Arthroplasty Register. Annual Report 2006. Available at: http://www.jru.orthop.gu.se. Accessed December 1, 2008.

36. Van den Donk S, Buma P, Slooff TJ, Gardeniers JW, Schreurs BW. Incorporation of morselized bone grafts: a study of 24 acetabular biopsy specimens. Clin Orthop Relat Res. 2002;396: 131-141.

37. Wan Z, Boutary M, Dorr LD. The influence of acetabular component position on wear in total hip arthroplasty. $J$ Arthroplasty. 2008;23:51-56.

38. Zahiri CA, Schmalzried TP, Szuszczewicz ES, Amstutz HC. Assessing activity in joint replacement patients. J Arthroplasty. 1998;13:890-895. 